\title{
LA INFLUENCIA DEL ACCESO AL USO DE COMPUTADORAS EN EDAD TEMPRANA SOBRE EL RENDIMIENTO EN MATEMÁTICAS
}

\author{
Andrés Fernández Aráuz ${ }^{1}$
}

Recibido: 22/07/2014

Aprobado: 09/09/2014

\begin{abstract}
RESUMEN
¿El acceso al uso de computadoras en edades tempranas tiene impacto sobre el rendimiento educativo de los estudiantes? La evidencia empírica de diseños experimentales muestra poca o nula relación entre el uso de las TIC's y el rendimiento académico, evaluando los resultados a uno o dos años plazo, pero con la base de datos de la evaluación PISA se puede analizar esta asociación varios años después de que el estudiante tuvo acceso a una computadora por primera vez.

Realizando un análisis exploratorio de los datos de los estudiantes costarricenses en la evaluación matemática de PISA 2012, y mediante la estimación de un modelo de regresión lineal, se logra comprobar que incluso controlando por factores con alto poder explicativo del rendimiento académico, la edad a la que se tuvo acceso a una computadora por primera vez sí es un determinante del rendimiento educativo.
\end{abstract}

PALABRAS CLAVES: PISA, RENDIMIENTO ACADÉMICO, EDUCACIÓN, TECNOLOGÍAS DE INFORMACIÓN Y COMUNICACIÓN (TIC'S).

\section{ABSTRACT}

Does early access to computer use improve student achievement? Empirical evidence from experimental designs shows little or no relationship between the use of ICT and academic performance. Using the data collected in PISA it can be analyzed this association some years after that the student had access to a computer for the first time.

Performing an exploratory data analysis of Costa Rican students in PISA 2012 mathematics assessment and by estimating a linear regression model even controlling for factors with high explanatory power on academic performance, it's shown that age at access to a computer is a determinant of educational performance.

KEY WORDS: PISA, ACADEMIC ACHIEVEMENT, EDUCATION, INFORMATION AND COMMUNICATION TECHNOLOGY (ICT).

$1 \quad$ Ministerio de Educación Pública, Departamento de Análisis Estadístico. Código postal 10102. San José Costa Rica; affa17@gmail.com 


\section{INTRODUCCIÓN}

En la última década, los principales indicadores educativos de Costa Rica se han caracterizado por mostrar mejoras en los referentes a acceso y permanencia en el sistema educativo, sin que necesariamente esto se traduzca en una mejora en la calidad educativa.

Si bien se han dado avances en el aumento en la cobertura $y$ disminución de la deserción (exclusión), persisten algunas brechas en el acceso a recursos educativos, especialmente a las tecnologías de información y comunicación ${ }^{2}$.

Según datos de la Fundación Omar Dengo, el Programa Nacional de Informática Educativa que comenzó en el año 1988 alcanzó en ese año a poco menos del $10 \%$ de la población estudiantil, llegando a 35\% en el año 2000 y a $70 \%$ en el año $2013^{3}$.

Sin embargo, aún no ha sido evaluado si esta cobertura ha beneficiado a todos los jóvenes en edades tempranas del desarrollo o si han tenido un impacto en el rendimiento académico de los estudiantes.

Utilizando una base de datos novedosa para el análisis del contexto educativo del país, esta investigación pretende responder a la siguiente pregunta: ¿el acceso al uso de computadoras en edades tempranas tienen impacto sobre el rendimiento educativo de los estudiantes?

El artículo se estructura de la siguiente manera: en la segunda parte se realiza una breve revisión de la literatura y de factores

$2 \quad$ La tasa neta de escolaridad en secundaria ha pasado de $52 \%$ en el año 2000 a $70 \%$ en el año 2013 y la deserción en ese nivel ha pasado de $12,4 \%$ en el 2001 a 9,9\% en el 2013 (Ministerio de Educación Pública (MEP), 2013).

El Programa Nacional de Informática Educativa (PRONIE) fue creado en 1988 en un esfuerzo conjunto del Ministerio de Educación Pública de Costa Rica (MEP) y la Fundación Omar Dengo (FOD). Su misión ha sido contribuir con el desarrollo de las capacidades de los estudiantes, a través de propuestas pedagógicas innovadoras apoyadas en las tecnologías digitales, concebidas como herramientas de aprendizaje. Actualmente cubre a centros educativos de primaria y de secundaria de todo el país. asociados al rendimiento; en la tercera parte se muestra la metodología del modelo utilizado para analizar la influencia de la edad del uso de las computadoras sobre el rendimiento académico, en la cuarta parte se presentan los resultados y en la última parte se muestran las conclusiones.

\section{MARCO TEÓRICO}

Tic's y educación

Usualmente, las políticas educativas se centran en proveer factores fácilmente cuantificables en los sistemas educativos, como dinero, infraestructura, equipo y mobiliario, libros, entre otros.

Estas políticas son las más usadas porque son fáciles de administrar y son visibles a la sociedad en general, por lo que son políticamente viables. Pero mejorar o incrementar la dotación de estos factores no se traduce necesariamente en una mejora en el proceso de aprendizaje de los estudiantes.

Sin embargo, el impacto de los recursos en el aprendizaje de los estudiantes puede ser limitado porque estos no necesariamente son colocados con el propósito de mejorar el aprendizaje del estudiante. Los centros educativos $y$ los sistemas escolares son lugares altamente politizados donde las decisiones son tomadas por un número de razones, de las cuales mejorar el aprendizaje de los estudiantes puede ser solo una. (Vegas \&Petrow, 2007, pág. 6)

Una de las variables que ha tomado relevancia en estudios recientes sobre el impacto de diferentes factores en el rendimiento académico es el acceso y aprendizaje de las tecnologías de información y comunicación (TICs), ya que estas:

"[...] tienen el potencial de mejorar el aprendizaje de los estudiantes. [...] Como las TICs están cada vez más presentes en la vida cotidiana y en el trabajo, los conocimientos computacionales se han convertido en un requisito básico para muchos trabajos". (Ídem) 
Así, muchos países han optado por políticas educativas en la que se destinan una mayor cantidad de recursos económicos para instalar o incrementar el equipamiento tecnológico de los centros educativos, ya que:

"Invertir en TICs es visto en el plano educativo como un camino primario para preparar a las personas a las nuevas demandas del mercado laboral $y, a$ la vez permitir que las poblaciones más vulnerables puedan desarrollar las competencias necesarias para tener igualdad de acceso a oportunidades de empleo". (Urzúa \&Veramendi, 2011, pág. 119).

Según la CEPAL, el acceso que los jóvenes puedan tener a las tecnologías de información y comunicación es el componente que ha tenido mayor desarrollo entre las políticas implementadas en América Latina:

"En efecto, se han hecho significativas inversiones en la instalación de infraestructura y equipamiento tecnológico en las escuelas, lo que ha facilitado que estas se conviertan en una puerta de entrada al mundo digital para amplios sectores de la población. La gran deuda, que plantea un gran desafío, es ahora incorporar las tecnologías digitales a los ambientes de aprendizaje y las instituciones educacionales, lo que trasciende aspectos puramente técnicos e involucra diversas variables, como las metodologías pedagógicas y los contenidos o materiales curriculares." (Bárcena, Prado, Hopenhayn y Pérez, 2014).

\section{Revisión de la literatura}

La literatura referente al impacto de las TIC's en las variables educativas se ha concentrado en estudiar particularmente si la introducción de estas tecnologías puede mejorar el aprendizaje. Sin embargo, estudios rigurosos han encontrado que el impacto de las TIC's sobre el rendimiento educativo es limitado o prácticamente nulo.
Cristia, Czerwonko, y Garofalo, (2010) evalúan la hipótesis de si el incremento en el acceso a las TIC's puede aumentar los años de educación completos en Perú, mostrando evidencia sobre el impacto del incremento al acceso de las computadoras en centros educativos de secundaria sobre las tasas de deserción y la matrícula inicial.

Para hacer esto, evalúan el impacto de la implementación de un programa educativo financiado por el Banco Interamericano de Desarrollo en el que se distribuyeron 10 computadoras a cada uno de 350 centros educativos públicos de secundaria en el año 2004 y lo comparan contra un grupo de centros educativos que habían recibido previamente computadoras en gobiernos anteriores $y$ que por lo tanto no eran elegibles para la participación en el programa. Concluyen que no hay evidencia de que la política tuviera un impacto significativo sobre las variables analizadas.

En un estudio similar realizado también en Perú, Cristia, Ibarrarán, Cueto, Santiago y Severín (2012) evalúan en un experimento aleatorio a gran escala otro programa que ha tenido gran auge a nivel mundial llamado Una Computadora por Niño (One Laptop Per Child, OLPC). Este programa busca mejorar el aprendizaje en las regiones más pobres del mundo suministrando computadoras personales a los niños para utilizar tanto en la escuela como en el hogar.

El programa OLPC fue implementado en Perú en el año 2008 con la distribución de 40.000 laptops en cerca de 500 centros educativos en regiones pobres, pero priorizando las que tuvieran electricidad $y$ acceso a Internet. Concluyen que la implementación del programa tuvo efectos limitados sobre el rendimiento escolar de los niños, pero impactos positivos en las habilidades cognitivas y competencias relacionadas con el uso de las computadoras.

Fairlie y Robinson (2012) realizan un experimento aleatorio, con una muestra de 1.123 estudiantes sobre 15 escuelas diferentes en cinco distritos en California, Estados Unidos, a los cuales se les asignó una computadora para utilizar en su hogar; concluyen que el uso de computadoras en el hogar por parte de estos 
estudiantes no tuvo ningún efecto (ni positivo ni negativo) sobre las variables analizadas, incluido el rendimiento medido a través de una prueba estandarizada:

\section{"Encontramos que las computadoras} para utilizar en el hogar incrementaron el uso total de las computadoras para trabajos escolares, pero también para juegos y otros entretenimientos. No encontramos evidencia de efectos positivos sobre otros factores, como el tiempo dedicado a ensayos, ayudar en la realización de tareas o asignaciones, el uso de software o incrementar las habilidades y el conocimiento computacional". (Fairlie\& Robinson, 2012, pág 23).

Otros estudios han analizado este mismo tema, llegando en la mayoría de los casos a las mismas conclusiones: el acceso y uso de computadoras tiene poco o nulo impacto sobre el rendimiento académico de los estudiantes (véase Angrist y Lavy (2002), Goolsbee y Guryan (2006), Barrera-Osorio (2009) y Linden (2008)).

No obstante, estas investigaciones (usualmente diseños experimentales) analizan el impacto inmediato de la política, a uno o dos años plazo, por lo costoso y complicado que sería evaluar el impacto de la política en un tiempo mayor, como 5,8 o 10 años.

\section{Determinantes del rendimiento educativo}

En el campo de la economía de la educación, el estudio de la variabilidad en el rendimiento educativo usualmente se concentra en el análisis multifactor, donde se estudia el impacto de varias variables a la vez sobre las diferencias en rendimiento educativo.

Para Costa Rica, en el año 2013 fueron publicadas dos investigaciones que estudiaron la relación entre el rendimiento de los estudiantes en la prueba PISA 2009+ y varias variables, en ambos casos concluyeron que son los factores asociados con el contexto familiar y personal del estudiante, los que más tienen impacto sobre el rendimiento educativo (Fernández \& Del Valle, 2013, Montero, Rojas, Zamora, y Rodino, 2013).
Los resultados de estas dos investigaciones son considerados en la estimación del modelo de regresión al momento de definir las variables de control. Sin embargo, el interés de este estudio no es replicar el modelo de función de producción educativa, que involucra varias variables, ya que actualmente se disponen de análisis actualizados en este sentido con bases de datos de PISA, y especialmente tomando en cuenta que la prueba PISA $2009+$ se realizó en el año 2010 mientras que los datos utilizados en esta investigación provienen de la prueba aplicada en el 2012, por lo que no es esperable que hayan cambios significativos en la dirección y magnitud de los resultados.

Sin embargo, los análisis realizados con la base de datos de PISA 2009+ no disponían de la variable de interés de este artículo, ya que en esa ocasión Costa Rica no tomó el cuestionario de familiaridad de los estudiantes a las tecnologías de información y comunicación; de ahí parte el interés por estudiar la importancia o no de esta variable.

En esta investigación se plantea la realización de un análisis exploratorio y de un modelo de regresión lineal para analizar la influencia del acceso en edad temprana de los estudiantes costarricenses al uso de una computadora sobre el rendimiento académico, con datos provenientes de la prueba de matemáticas de la evaluación internacional PISA 2012.

\section{METODOLOGÍA}

Para poder determinar si la asociación entre la edad de uso de una computadora por primera vez y el rendimiento académico es realmente significativa se recurre a la estimación de una regresión lineal.

Se utilizará como variable dependiente el resultado de los estudiantes en la prueba de matemáticas ${ }^{4}$, y como variables independientes una matriz de variables de control $(X)$ y la variable de interés.

De esta forma, el modelo a estimar toma la siguiente forma:

$4 \quad$ PISA provee cinco valores plausibles del rendimiento de los estudiantes en lugar de una única medida de resultado para cada una de las áreas evaluadas, es decir, cinco valores plausibles para lectura, cinco valores plausibles para matemática $y$ cinco valores plausibles para ciencias. En esta investigación únicamente fueron utilizados los valores plausibles de la evaluación de matemáticas. 


$$
P V_{i}=\beta_{0}+\beta_{1} X_{i}+\beta_{2} \text { Seis }_{i}+\beta_{3} \text { Siete }_{i}
$$

Donde $P V_{i}$ es el valor plausible o resultado del individuo "i" en la evaluación de matemáticas, $X_{i}$ es una matriz de variables de control, que incluye al Índice de Estatus Económico, Social y Cultural $\left(\operatorname{ESCS}^{5}\right)$, el sexo del individuo, la condición de repitencia (formado por dos variables dicotómicas que resumen la información de si el individuo repitió un grado en escuela o un grado en III Ciclo de colegio), Seis $_{i}$ es una variable dicotómica que toma el valor de uno si el individuo usó por primera vez una computadora a los seis años o antes, es una variable dicotómica que toma el valor de uno si el individuo usó por primera vez una computadora entre los siete $y$ ocho años de edad, y $\beta_{i}$ es el coeficiente que acompaña a cada variable.

El modelo estima el impacto de las variables relacionadas con el uso temprano de una computadora en el rendimiento académico; controlando otros factores que han demostrado tener efecto sobre el rendimiento (estatus socioeconómico, sexo, repitencia) ${ }^{6}$.

Es importante reseñar nuevamente que el análisis de relación entre los factores familiares $y$ personales, los factores escolares $y$ los factores institucionales sobre el rendimiento educativo ha sido estudiado recientemente

5 Para una explicación más detallada sobre el análisis de componentes principales utilizado en el cálculo del índice de Estatus Económico, Social y Cultural, así como del proceso de ponderación utilizado para cada país puede consultar OECD, (2012).

La matriz de correlaciones muestra la mayor correlación entre el estatus socioeconómico y el rendimiento $(0,41)$ y en segundo lugar entre el estatus socioeconómico y la variable de edad de uso de la computadora antes de los seis años $(0,30)$; sin embargo, esta correlación no es tan alta como para evidenciar una posible multicolinealidad entre los factores. Para comprobar esta hipótesis, se procedió al cálculo del factor de inflación de la varianza, el cual resultó mucho menor a 10 en todos los casos, por lo tanto se concluye que no se presentan problemas de multicolinealidad. La correlación entre la variable de interés y el rendimiento es de 0,25 . para el caso de Costa Rica con la base de datos de PISA 2009+ (Fernández y Del Valle, 2013), por lo que la finalidad de este artículo no es la reestimación de la función de producción educativa, sino enfocarse en el impacto de una variable que no pudo ser incorporada en los modelos de regresión que usaron los datos de PISA 2009+, y que puede aportar resultados interesantes para la definición de políticas educativas enfocadas en tecnologías de información y comunicación.

Además del análisis de regresión en la siguiente sección de resultados se realiza un análisis exploratorio de los datos.

\section{RESULTADOS}

\section{El Programa PISA y el acceso a computadoras}

El Programa Internacional de Evaluación de Estudiantes (PISA- Programmefor International StudentAssessment) es un esfuerzo conjunto entre los países miembros de la OCDE que busca medir la capacidad que tienen los estudiantes en edad de 15 años y que se acercan a concluir el ciclo de escolaridad obligatoria, para afrontar los retos de la sociedad actual ${ }^{7}$. Por lo tanto, según la Organización para la Cooperación y el Desarrollo Económico (OECD), PISA evalúa:

"El grado en el que los estudiantes pueden utilizar sus habilidades en lectura para comprender e interpretar los diversos tipos de material escrito a los que se encontrarán expuestos a lo largo de su vida; el grado en el que estos estudiantes pueden usar sus habilidades y conocimientos matemáticos para resolver diferentes retos y problemas numéricos

\footnotetext{
$7 \quad$ La muestra de PISA está diseñada sobre una población de estudiantes con edades entre los 15 años $y$ tres meses (completos) y 16 años $y$ dos meses (completos) que asisten a las instituciones educativas oficiales y se encuentran cursando un grado equivalente al séptimo o superior para todos los países participantes. Por conveniencia, se hace referencia a la población como estudiantes de 15 años. El diseño consiste en una muestra aleatoria estratificada de conglomerados.
} 
y espaciales; el grado en que los estudiantes pueden utilizar sus habilidades $y$ conocimientos científicos para entender, interpretar y resolver varios tipos de retos y situaciones científicas". (OECD, 2012)

En el año 2012, Costa Rica participó por segunda vez en esta evaluación, obteniendo los mismos resultados que los vistos en el año 2010 (es decir, el rendimiento promedio de los estudiantes costarricenses de 15 años no varió del año 2010 al 2012), ubicándose en los puestos 56, 49 y 51 de 65 países en las evaluaciones de matemáticas, lectura y ciencias respectivamente.

No obstante, más allá de este ranking general, las evaluaciones de PISA proveen información valiosa sobre características de los estudiantes $y$ sus sistemas educativos, que al ser analizadas con cautela pueden aportar en el estudio de las desigualdades educativas que se encuentran presentes en un país.

Además de la medición de habilidades y conocimientos de los estudiantes mediante una prueba escrita, PISA también realiza cuestionarios para recolectar información que permita analizar y contextualizar adecuadamente los resultados de las pruebas. Se aplican cuestionarios a los estudiantes para obtener información sobre aspectos del hogar, familia, su centro educativo $y$ sus intereses $y$ gustos propios en temas educativos y no educativos, $y$ un cuestionario sobre familiaridad de los estudiantes a las tecnologías de información y comunicación ${ }^{8}$.

En este cuestionario, una de las preguntas a la que respondieron los estudiantes fue: ¿Qué edad tenía cuándo usó una computadora por primera vez? Las opciones fueron: 6 años o menos, 7 a 9 años, 10 a 12 años, 13 años o más. Los resultados fueron los siguientes:

Un 27\% de los estudiantes afirma haber tenido acceso a una computadora por primera vez antes de cumplir los seis años, mientras que un $32 \%$ de los estudiantes tuvo acceso por primera vez a una computadora después de los 10 años de edad. La relación con el rendimiento mostrado en la prueba PISA es directa: entre más temprano se haya tenido acceso a una computadora mayor es el rendimiento mostrado en la prueba (ver cuadro 1$)^{9}$ :

8 Otros cuestionarios son administrados a los directores de los centros educativos y si el país así lo solicita se aplican cuestionarios a los padres de familia.

$9 \quad$ Todos los promedios presentados en este artículo corresponden a promedios ponderados en los que se utilizó la Ponderación Final del Estudiante (W_ FSTUWT) de las bases de datos de PISA. Para el cálculo de los errores estándar se utiliza la técnica de Replicación Repetida y Equilibrada (BRR), utilizando otros 80 ponderadores (W_FSTR1, W_FSTR2..., W_FSTR80) siguiendo los capítulos 7 y 8 del Manual de Análisis de Datos de PISA (OECD, 2009).

CUADRO 1

RELACIÓN ENTRE LA EDAD A LA QUE USÓ COMPUTADORA POR PRIMERA VEZ Y EL RENDIMIENTO ACADÉMICO

\begin{tabular}{|c|c|c|c|c|c|c|c|c|}
\hline \multirow{3}{*}{$\begin{array}{c}\text { Edad } \\
6 \text { o menos }\end{array}$} & \multirow{2}{*}{\multicolumn{2}{|c|}{$\begin{array}{c}\text { Proporción } \\
\text { (en porcentaje) }\end{array}$}} & \multicolumn{6}{|c|}{ Rendimiento promedio } \\
\hline & & & \multicolumn{2}{|c|}{ Lectura } & \multicolumn{2}{|c|}{ Matemáticas } & \multicolumn{2}{|c|}{ Ciencias } \\
\hline & 27,1 & $(1.0)$ & 468 & $(4.3)$ & 437 & $(4.1)$ & 458 & (3.7) \\
\hline 7 а 9 & 35,4 & $(1.0)$ & 448 & $(3.2)$ & 413 & $(2.6)$ & 439 & (2.8) \\
\hline 10 a 12 & 21,7 & $(0.9)$ & 428 & $(4.5)$ & 393 & $(3.8)$ & 418 & $(4.1)$ \\
\hline 13 o más & 11,0 & $(0.9)$ & 402 & $(5.3)$ & 366 & $(3.5)$ & 389 & $(4.6)$ \\
\hline Total país & \multicolumn{2}{|c|}{100} & 441 & $(3.5)$ & 407 & $(3.0)$ & 429 & (2.9) \\
\hline
\end{tabular}

El error estándar se muestra entre paréntesis.

Fuente: Cálculos propios con datos de PISA 2012.

La suma de la proporción no es 100 . La diferencia se debe a la no respuesta. 
Esta relación es importante, porque muestra asociación entre la edad de acceso a la computadora y un rendimiento académico posterior, especialmente porque las diferencias en rendimiento promedio entre grupos de edad sí son estadísticamente significativas para las tres áreas evaluadas en PISA 2012.

En los siguientes párrafos se describirán algunas de las principales características de los estudiantes según los grupos de edad de acceso por primera vez a una computadora, con el objetivo de evaluar posibles correlaciones con otras variables que pudieran incidir total o parcialmente en las diferencias en rendimiento.

\section{Características de los estudiantes}

El hecho de que un estudiante tenga acceso a una computadora a una edad temprana se encuentra relacionado de manera directa con el poder adquisitivo de la familia: para los que tuvieron acceso a una computadora antes de los 6 años de edad el 10\% se ubica en el cuartil de más bajo estatus socioeconómico y casi la mitad se ubican en el cuartil más alto; caso contrario ocurre con los que comenzaron a utilizar una computadora a edades tardías (13 o más), donde más de la mitad de los estudiantes se ubica en el cuartil más bajo y menos del $3 \%$ en el cuartil de estatus más alto (ver gráfico 1$)^{10}$.

$10 \quad$ Cuartiles calculados con base en el Índice de Estatus Económico, Social y Cultural. Para una explicación más detallada de este índice consulte el reporte técnico de PISA (OECD, 2012).

\section{GRÁFICO 1}

PROPORCIÓN DE ESTUDIANTES EN CADA CUARTIL DE ESTATUS SOCIOECONÓMICO SEGÚN EDAD DE USO DE LA COMPUTADORA POR PRIMERA VEZ

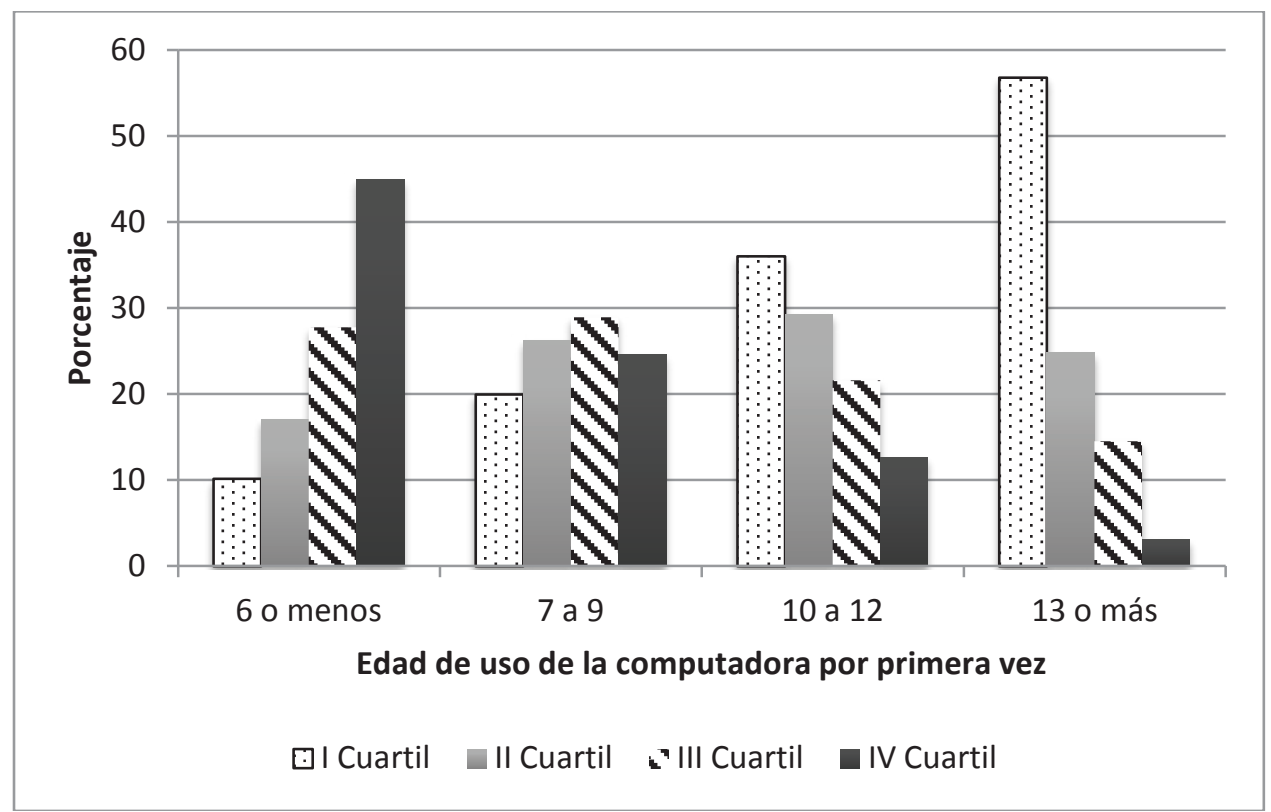

Fuente: Elaboración propia con datos de la evaluación PISA 2012. 
Además, estos estudiantes presentan algunas características que también pueden influir en el mayor rendimiento mostrado en las pruebas: la mayoría de los estudiantes que tuvieron acceso a una computadora antes de los seis años, también comenzaron el ciclo educativo más temprano, asistiendo a la educación preescolar (91\% vs $67 \%)$.
Por otra parte, la proporción de estudiantes que han repetido uno o más años en escuela o en colegio son menores para el grupo de estudiantes que usó una computadora antes de los seis años, es decir, los estudiantes que tuvieron acceso a una computadora a edades tardías han repetido años escolares en mayor medida que los otros educandos (ver cuadro 2 ).

CUADRO 2

CARACTERÍSTICAS DE LOS ESTUDIANTES PARTICIPANTES EN PISA 2012 SEGÚN EDAD DE USO DE LA COMPUTADORA POR PRIMERA VEZ (EN PORCENTAJES)

\begin{tabular}{lcccc}
\hline \multirow{2}{*}{ Característica } & \multicolumn{4}{c}{ Edad a la que usó computadora por primera vez } \\
\cline { 2 - 5 } & 6 o menos & 7 a 9 & 10 a 12 & 13 o más \\
\hline Mujer & 51 & 53 & 53 & 64 \\
Colegio Público & 73 & 85 & 94 & 98 \\
Asistió a preescolar & 91 & 86 & 15 & 67 \\
Repitió grado en escuela & 9 & 23 & 22 & 25 \\
Repitió grado en colegio & 18 & & & 25 \\
\hline
\end{tabular}

Fuente: Cálculos propios con datos de PISA 2012.

Cuartiles calculados con base en el índice de estatus económico, social y cultural (ESCS)

\section{Acceso a Internet por primera vez}

El hecho de que un niño tenga acceso al uso de una computadora no significa necesariamente que también tenga acceso a Internet.
Si bien entre menor sea la edad a la que se haya tenido acceso a una computadora por primera vez, también menor es la edad a la que se tuvo acceso a Internet por primera vez, existe un rezago entre ambos (ver cuadro 3 ).

CUADRO 3

RELACIÓN ENTRE EDAD DE USO DE LA COMPUTADORA POR PRIMERA VEZ Y ACCESO A INTERNET POR PRIMERA VEZ

\begin{tabular}{lcccc}
\hline \multirow{2}{*}{ Edad a la que usó Internet por primera vez } & \multicolumn{4}{c}{ Edad a la que usó computadora por primera vez } \\
\cline { 2 - 5 } & 6 o menos & 7 a 9 & 10 a 12 & 13 o más \\
\hline 6 o menos & 29,5 & 1,3 & 0,7 & 0,1 \\
7 a 9 & 42,3 & 42,3 & 3,4 & 0,5 \\
10 a 12 & 23,7 & 46,3 & 62,7 & 6,3 \\
13 o más & 4,1 & 9,1 & 32,0 & 88,7 \\
\hline Total & 99,6 & 99,0 & 98,8 & 95,6 \\
\hline
\end{tabular}

Fuente: Cálculos propios con datos de PISA 2012.

La suma de los porcentajes no es 100 debido a la no respuesta 
Solo el 30\% de los jóvenes que usaron una computadora a los seis años o antes tuvieron acceso a Internet en el mismo momento, mientras que un $42 \%$ tuvo acceso a internet algunos años después:

\section{Recursos tecnológicos en el hogar}

Como fue mostrado anteriormente, la mayoría de estudiantes que tuvieron acceso a una computadora por primera vez a los seis años de edad o antes pertenecen a los dos cuartiles más altos de estatus socioeconómico, mientras que los que usaron una computadora después de los 10 años, provienen en su mayoría de los dos cuartiles de estatus socioeconómico más bajos.

Esto repercute en el acceso que hoy día tienen los estudiantes en sus hogares a recursos tecnológicos y en el uso de los mismos. Por ejemplo, mientras que un $65 \%$ de los estudiantes que usaron una computadora a los seis años $o$ antes tienen una computadora de escritorio en su casa o una laptop y los utilizan, en el caso de los que tuvieron acceso tardío a una computadora esos porcentajes son de $41 \%$ y $33 \%$ respectivamente (ver cuadro 4).

\begin{tabular}{lcc}
\hline & Edad de uso de computadora por primera vez \\
\cline { 2 - 3 } Dispositivo & 6 o menos & 10 o más \\
\hline Computadora de escritorio & 65,1 & 41,4 \\
Laptop & 64,4 & 32,7 \\
Tablet & 19,4 & 4,6 \\
Internet & 82,6 & 51,6 \\
Consola de video juegos & 55,9 & 24,4 \\
Celular sin acceso a internet & 50,5 & 41,9 \\
Celular con acceso a internet & 75,3 & 63,3 \\
Reproductor de música & 65,7 & 26,4 \\
Impresora & 66,8 & 34,0 \\
Llave maya & 86,5 & 64,6 \\
\hline
\end{tabular}

Fuente: Cálculos propios con datos de PISA 2012.

El porcentaje corresponde a los estudiantes que tienen el dispositivo en su hogar y además lo utilizan

En el caso del Internet, el $83 \%$ de los estudiantes que utilizaron una computadora a una edad temprana lo tienen $y$ lo utilizan en su hogar, contra un $52 \%$ de los que accedieron a una computadora a los 10 años o posterior.

Esto último se ve reflejado en el tiempo que los estudiantes dedican a navegar por Internet en un día típico: mientras que una cuarta parte de los estudiantes que accedieron a una computadora después de los 10 años utilizan el Internet fuera de horario escolar por más de dos horas; poco más de la mitad de los que usaron una computadora antes de los seis años navegan por más de dos horas en Internet (ver cuadro 5). 
CUADRO 5

TIEMPO DIARIO DESTINADO POR LOS ESTUDIANTES AL USO DE INTERNET FUERA DEL COLEGIO (EN PORCENTAJES)

\begin{tabular}{lccccccc}
\hline $\begin{array}{l}\text { Edad de uso de la } \\
\text { computadora por } \\
\text { primera vez }\end{array}$ & Nada & 1 a 30 min & 30 a 60 min & 1 a 2 h & 2 a 4 h & 4 a 6 h & 6 h o más \\
\cline { 2 - 9 } & 5,5 & 11,5 & 9,7 & 23,2 & 24,5 & 14,2 & 10,9 \\
\hline o menos & 17,6 & 18,8 & 15,5 & 23,3 & 14,2 & 5,1 & 3,5 \\
\hline
\end{tabular}

Fuente: Cálculos propios con datos de PISA 2012.

Además, el uso que se le da a la computadora no varía entre grupos, pero sí su intensidad de uso: por ejemplo, en ambos casos la computadora es más utilizada para conectarse a redes sociales como Facebook, navegar por diversión y descargar música, películas y juegos, pero estas actividades son realizadas con mayor frecuencia por los que tuvieron acceso a una computadora a los seis años de edad o antes (ver cuadro 6).

CUADRO 6

PORCENTAJE DE ESTUDIANTE QUE UTILIZAN LA COMPUTADORA PARA DIFERENTES ACTIVIDADES CASI TODOS O TODOS LOS DÍAS DE LA SEMANA SEGÚN EDAD DE ACCESO A UNA COMPUTADORA (EN PORCENTAJES)

\begin{tabular}{lcc}
\hline & Edad de uso de computadora por primera vez \\
\cline { 2 - 3 } Actividad & 6 o menos & 10 o más \\
\hline Jugar sólo & 23,7 & 11,1 \\
Jugar juegos colaborativos por Internet & 16,9 & 6,4 \\
Usar correo electrónico & 45,7 & 21,9 \\
Chatear (Skype...) & 48,6 & 22,7 \\
Redes sociales (Facebook, MySpace...) & 69,8 & 38,7 \\
Navegar por diversión (YouTube...) & 60,3 & 30,0 \\
Leer noticias & 24,1 & 10,3 \\
Obtener información práctica & 36,1 & 15,4 \\
Descargar música, películas.... & 56,2 & 29,2 \\
Cargar archivos propios para compartir & 22,4 & 12,6 \\
\hline
\end{tabular}

Fuente: Cálculos propios con datos de PISA 2012.

De manera similar, en cuanto a las actividades académicas, los principales usos de ambos grupos son hacer tareas en la computadora, buscar información en Internet para hacer los trabajos escolares y comunicarse con otros estudiantes vía correo electrónico, aunque estas actividades las realizan de una manera más intensa los que tuvieron acceso a una computadora a los seis años o antes (ver cuadro 7). 
CUADRO 7

PORCENTAJE DE ESTUDIANTE QUE UTILIZAN LA COMPUTADORA PARA ACTIVIDADES ESCOLARES AL MENOS UNA VEZ A LA SEMANA SEGÚN EDAD DE ACCESO A UNA COMPUTADORA (EN PORCENTAJES)

\begin{tabular}{lcc}
\hline & \multicolumn{2}{c}{$\begin{array}{c}\text { Edad de uso de computadora por } \\
\text { primera vez }\end{array}$} \\
\cline { 2 - 3 } Actividad & 6 o menos & 10 o más \\
\hline Buscar información en Internet para preparar un trabajo escolar & 73,9 & 52,0 \\
Usar el correo electrónico para comunicarse con otros estudiantes sobre trabajos escolares & 61,5 & 37,9 \\
Usar el correo electrónico para enviarle los trabajos y tareas a los profesores & 33,6 & 20,8 \\
Descargar o cargar material en el sitio web del colegio & 32,2 & 21,0 \\
Hacer tareas en la computadora & 76,6 & 52,4 \\
Compartir material escolar con otros estudiantes & 41,1 & 27,7 \\
\hline
\end{tabular}

Fuente: Cálculos propios con datos de PISA 2012

\section{El modelo de regresión}

Utilizando como variable dependiente el resultado de los estudiantes costarricenses en la evaluación de matemáticas de la prueba PISA 2012 , se obtuvo lo siguiente (ver cuadro 8) ${ }^{11}$ :

\section{CUADRO 8}

INTERVALOS AL 99\% DE CONFIANZA DE LOS COEFICIENTES DE LA REGRESIÓN LINEAL

\begin{tabular}{|c|c|c|c|}
\hline Variable & $\begin{array}{l}\text { Lím } \\
\text { Inferior }\end{array}$ & Estimado & $\begin{array}{c}\text { Lím } \\
\text { Superior }\end{array}$ \\
\hline Intercepto & 431,7 & 436,7 & 441,8 \\
\hline ESCS & 13,4 & 15,4 & 17,3 \\
\hline Sexo & $-29,3$ & $-25,0$ & $-20,7$ \\
\hline Repitió escuela & $-48,9$ & $-42,5$ & $-36,1$ \\
\hline Repitió colegio & $-39,0$ & $-33,8$ & $-28,6$ \\
\hline Seis $o$ antes & 19,5 & 25,3 & 31,1 \\
\hline Siete $y$ nueve & 8,0 & 13,0 & 18,1 \\
\hline \multicolumn{2}{|c|}{ R2 ajustado } & 0,31 & \\
\hline \multicolumn{2}{|c|}{ Observaciones } & 4571 & \\
\hline
\end{tabular}

Fuente: Cálculos propios con datos de PISA 2012.

Todas las variables son significativas al $99 \%$ de confianza

$11 \quad$ Para cada uno de los cinco valores plausibles de la evaluación de matemáticas se estimaron 81 regresiones, por lo que en total fueron estimadas 405 regresiones mediante mínimos cuadrados ponderados utilizando los 81 ponderadores de la base de datos de PISA.
Las variables incluidas en el modelo explican un $31 \%$ de la variabilidad en el rendimiento, lo cual es un valor muy cercano a otras estimaciones que han utilizado muchas otras variables $^{12}$.

Después de controlar por los factores mencionados, las variables de interés resultaron ser significativas para explicar el rendimiento académico de los estudiantes en la evaluación de matemática de PISA 2012.

Haber utilizado una computadora a los seis años o antes aumenta el rendimiento del estudiante en un valor entre 19 y 31 puntos, mientras que haberla utilizado entre los $7 y$ los nueve años incrementa el puntaje obtenido en un valor entre 8 y 18 puntos.

\section{CONCLUSIONES}

La participación de Costa Rica en la evaluación internacional PISA no es simplemente una herramienta más al alcance de los tomadores de decisiones de política educativa del país, sino que su alcance dista mucho del de otras estadísticas también útiles y preparadas en el país.

$\mathrm{Su}$ principal ventaja es que, al disponer de datos por estudiante en una muestra representativa del país, permite establecer y analizar

12 Véase Fernández y Del Valle, 2013; Fernández, A 2013. 
las relaciones de asociación entre diferentes características con el rendimiento mostrado por los estudiantes en las pruebas de matemáticas, lectura y ciencias.

Estudios previos habían mostrado que el estatus económico, social y cultural de los estudiantes es la variable que tiene el mayor peso en la explicación de la variabilidad en los resultados obtenidos en la evaluación PISA; otros factores, como el tipo de centro educativo (público o privado) influyen en menor medida en la explicación de las desigualdades en rendimiento.

Sin embargo, un factor que no puedo ser incorporado en los modelos de las investigaciones precedentes fue la pregunta que se intentó responder con este artículo: ¿influye la edad a la que se usa una computadora por primera vez en el rendimiento académico que posteriormente tienen los estudiantes? La literatura sobre este tema usualmente no logra demostrar causalidad entre el acceso a las tecnologías de información y comunicación y una mejora inmediata en el rendimiento académico.

No obstante, con los datos de la prueba PISA se puede probar esta hipótesis varios años después de que un estudiante tuvo su primer contacto con este tipo de tecnología.

Los resultados de la investigación demuestran que entre más temprano se tenga acceso a una computadora, mayor puede ser el rendimiento académico de los estudiantes, y que esta variable es significativa incluso controlando por el estatus socioeconómico de los estudiantes.

Este resultado es valioso desde el punto vista del diseño de política educativa, ya que sugiere que para disminuir en alguna medida la desigualdad en el rendimiento educativo es necesario poder dotar de tecnologías de información y comunicación a todos los niños, especialmente cuando comienzan la educación primaria.

Según el IV Informe del Estado de la Educación del Programa Estado de la Nación (2013) a pesar del avance en la cobertura de las TIC's en el sistema educativo, persisten brechas importantes, especialmente en las zonas alejadas del Valle Central.
Además, no se trata únicamente de la dotación de laboratorios, sino también del adecuado mantenimiento y la introducción de tecnologías digitales móviles y nuevos modelos educativos en el aula.

A diferencia de otros factores que han sido identificados como importantes para explicar la desigualdad educativa en rendimiento, la introducción de computadoras en edades tempranas es un instrumento que sí se encuentra al alcance del Ministerio de Educación, y que podría tener altos dividendos futuros si además es acompañado de una política educativa acorde con los nuevos modelos pedagógicos que incorporen estos recursos como una herramienta poderosa en el estudio y análisis de información.

\section{REFERENCIAS}

Angrist, J., \& Lavy, V. (2002). New evidence on classroom computers and pupil learning. The Economic Journal (October), 735-765.

Bárcena, A., Prado, A., Hopenhayn, M., \& Pérez, R. (2014). La integración de las tecnologías digitales en las escuelas de América Latina y el Caribe: Una mirada multidimensional. Libros de la CEPAL, 124. Naciones Unidas.

Cristia, J., Czerwonko, A., \& Garofalo, P. (2010). Does ICT Increase Years of Education? Evidence from Peru. Washington D.C.: Office of Evaluation and Oversight. Working Paper: OVE/WP-01/10. InterAmerican Development Bank.

Cristia, P., Ibarrarán, P., Cueto, S., Santiago, A., \& Severín, E. (2012). Technology and Child Development: Evidence from the One Laptop per Child Program. Deparment of Research and Chief Economist. IDB working paper series $\mathrm{N}^{\circ}$ 304. Inter-American Development Bank.

Fairlie, R., \& Robinson, J. (2012). Experimental Evidence on the effects of Home Computers on Academic Achievement among Schoolchildren.University of California.

Fernández, A. (2013). Análisis de la Resiliencia Educativa de los Estudiantes 
Costarricenses con datos de la Prueba de Lectura de la Evaluación PISA 2009. Revista de Ciencias Económicas Vol 31 $\mathrm{N}^{\circ}$ 2. ISSN: 0252-9521. Universidad de Costa Rica.

Fernández, A., \& Del Valle, R. (2013). Desigualdad Educativa en Costa Rica: La brecha entre los estudiantes de colegios públicos y colegios privados. Revista CEPAL No 111.

Goolsbee, A., \& Guryan, J. (2006). The impact of internet subsidies in public schools. Review or Economics and Statistics 88, 336-347. doi: 10.1162/rest.88.2.336.

Linden, L. (2008). Complement or Substitute? The effect of technology on Student Achievement in India.Columbia University, MIT Jameel Poverty Action Lab, IZA.

Ministerio de Educación Pública. (2013). Expansión del Sistema Educativo Costarricense, 2013. Publicación $\mathrm{N}^{\circ} 344-$ 13. SSN-1409-0422. Departamento de Análisis Estadístico. MEP.

Montero, E., Rojas, S., Zamora, E., \& Rodino, A. (2013). Costa Rica en las pruebas PISA 2009 de Competencia Lectora y Alfabetización Matemática. Cuarto
Informe del Estado de la Educación: Programa Estado de La Nación.

Organización para la Cooperación y el Desarrollo Económico. (2009). PISA Data Analysis Manual. SPSS Second Edition.OECD Publishing. do.org/10.1787/9789264056275-en.

Organización para la Cooperación y el Desarrollo Económico. (2012). PISA 2009 Technical Report. OECD Publishing. doi. org/10.1787/9789264091504-en.

Programa Estado de la Nación. (2013). Cuarto Informe del Estado de la Educación. San José, Costa Rica: Programa Estado de la Nación en Desarrollo Humano Sostenible. $4^{\circ}$ edición.

Urzúa, S., \& Veramendi, G. (2011). The impact of Out-of-Home Child Care Centers on Early Childhood Development. Deparment of Research and Chief Economist, Working Paper Series $\mathrm{N}^{\circ}$ IDB-WP-240. Inter-American Development Bank.

Vegas, E., \& Petrow, J. (2007). Raising Student Achievement in Latin America: The Challenge for the 21st Century. Washington, D.C.: Latin American Development Forum. Banco Mundial. 
